\title{
ON THE DIFFERENTIABILITY OF AFFINE AND PROJECTIVE TRANSFORMATIONS ${ }^{1}$
}

\author{
F. BRICKELL
}

1. Introduction. Let $M$ denote a differentiable manifold of dimension $d$ and suppose that a differentiable symmetric affine connection is given on $M$, where the word differentiable implies differentiability of class $C^{\infty}$. Unless a particular class is specified this implication is to be understood throughout the paper. Let $\phi$ denote a diffeomorphism of $M$ onto itself; then it is usual in differential geometry to define $\phi$ to be an affine transformation, or a projective transformation, according as $\phi$ preserves the geodesics of the affine connection as parametrized curves, or as point sets. These definitions still make sense if $\phi$ is not assumed to be a diffeomorphism but merely a one-to-one transformation of $M$ onto itself, and it is natural to ask whether the definitions then imply the differentiability of $\phi$. We show that this is true under the stronger assumption that $\phi$ is a homeomorphism, and with the restriction $d>2$ for projective transformations. Our theorems are formulated and proved for a general system of paths [1], which is a more general structure than the system of geodesics of an affine connection, but the proof of the theorem concerning projective transformations is restricted to the reversible case. The author wishes to thank Professor H. C. Wang for suggesting that some such extension of the fundamental theorem of real projective geometry might be possible.

2. Definition and properties of a general system of paths. We give a global definition of a system of paths on $M$ based on a slight modification of the notion of a spray [2, p. 67]. Let $T(M)$ denote the space of tangent vectors to $M, T^{\prime}(M)$ the open submanifold of nonzero tangent vectors, and $\pi$ the projection of $T(M)$ onto $M$. We also identify a real number $s$ with the mapping $X \rightarrow s X$ of $T(M)$ into itself and denote the differentials of the mappings $\pi, s$ by $\pi_{*}, s_{*}$. Now suppose that $\xi$ is a differentiable vector field on $T^{\prime}(M)$ satisfying the conditions

(i) $\pi_{*} \xi(X)=X$,

(ii) $\xi(s X)=s_{*} s \xi(X)$ for $s>0$,

and extend $\xi$ to $T(M)$ by defining it to be zero on zero vectors. Then a path on $M$ is a differentiable curve whose natural lift to $T(M)$ is an

Received by the editors June, 22, 1964.

${ }^{1}$ This research was supported by the National Science Foundation under grant G 24154 . 
integral curve of $\xi$. The totality of paths (for a given $\xi$ ) is called a system of paths on $M$; it is not difficult to see that this definition agrees with the one given in [1]. If condition (ii) is also satisfied for $s<0$ then the system of paths is said to be reversible; in this case $\xi$ is a spray. We remark that the extension of $\xi$ to $T(M)$ is, in general, of class $C^{1}$ only.

For $X \in T(M)$ denote by $\beta_{X}$ the unique integral curve of $\xi$ with $\beta_{X}(0)=X$, and let $D$ be the set of vectors $X$ such that $\beta_{X}(1)$ is defined. The mapping $D \rightarrow M$ defined by

$$
X \rightarrow \pi \beta_{X}(1)
$$

is called the exponential map and is denoted by exp. It follows from [2, Chapter IV] that $D$ is open, and that the exponential map is of class $C^{1}$ in general and of class $C^{\infty}$ on $D \cap T^{\prime}(M)$. The restriction of exp to the tangent space $T_{x}$ at $x \in M$ is denoted by $\exp _{x}$. It follows as in [2, Chapter IV, §4] that $\exp _{x}$ is a local diffeomorphism at the zero vector of class $C^{1}$ in general, and of class $C^{\infty}$ on nonzero vectors. The paths through $x$ are just the curves $t \rightarrow \exp _{x} t X$ for $X \in T_{x}$.

We now give several definitions. A neighborhood $V$ of the zero vector in $T_{x}$ is star-shaped if, for each $X \in V, t X$ is also $\in V$ for $0 \leqq t \leqq 1$. A normal neighborhood of $x \in M$ is a neighborhood $\exp _{x} V$ where $\exp _{x}$ is a diffeomorphism on $V$ and $V$ is star-shaped. A neighborhood $U$ on $M$ is simple if, for any ordered pair of points $y, z$ in $U$, there exists at most one path from $y$ to $z$ lying entirely in $U$. This is to exclude paths obtained by affine changes of parameter $t^{\prime}=\alpha t+\beta$ with $\alpha>0$. A neighborhood $U$ on $M$ is convex if any ordered pair of points $y, z$ in $U$ can be joined by a path lying entirely in $U$.

Let $x$ be any point of $M$; our proofs will use a neighborhood of $x$ which is simple, convex and contained in a normal neighborhood of each of its points. We call such a neighborhood a $C$-neighborhood; the existence of such neighborhoods follows from the "fundamental lemma" and Theorem 1 of [3].

3. The differentiability of affine transformations. We need the following lemma concerning a system of paths on a differentiable manifold $M$.

Lemma 3.1. Consider those positive values of $t$ on two paths $a(t)$ $=\exp _{x} t A, b(t)=\exp _{x} t B$ for which $a(t)$ and $b(t)$ lie in a $C$-neighborhood $U$ of $x$. Parametrize the unique path in $U$ from $a(t)$ to $b(t)$ so that $a(t)$ and $b(t)$ have parameter values 0 and 1 respectively, let $c(s, t)$ be the point on this path of parameter value $s(0 \leqq s \leqq 1)$, and put $c(s, t)$ $=\exp _{x} t C(s, t)$. Then 


$$
\lim _{t \rightarrow 0} C(s, t)=(1-s) A+s B .
$$

Proof. We consider the local mapping $\exp _{x}^{-1} \exp$ of $T(M)$ into $T_{x}$. Using a local differentiable section $\left\{e_{1} \cdots e_{d}\right\}$ of the frame bundle over $M$ we identify $T(U)$ with $U \times R^{d}$ and write the mapping as $Y=\psi(y, X)$, where $X$ and $Y$ are vectors in $R^{d}, y \in U$ and $\psi$ is differentiable of class $C^{1}$ at least. Denote by $\psi^{\prime}(y, X)$ the derivative of the mapping $X \rightarrow \psi(y, X)$ of $R^{d}$ in to itself, this derivative being defined as in $[2$, Chapter $1, \S 3]$. Since $\psi(x, X)=X$ we have $\psi^{\prime}(x, X)=I$ where $I$ denotes the identity transformation. We define a vector $L(t)$ by $\exp _{a(t)} L(t)=b(t)$ so that $\exp _{a(t)} s L(t)=c(s, t)$, and we obtain the equations

$$
t A=\psi(a(t), 0), \quad t C(s, t)=\psi(a(t), s L(t)), \quad t B=\psi(a(t), L(t)) .
$$

Thus, with the standard norm in $R^{d}$,

$$
\begin{aligned}
\|t B-t A-L(t)\| \leqq & \left\|t B-t A-\psi^{\prime}(a(t), 0) L(t)\right\| \\
& +\left\|\psi^{\prime}(a(t), 0)-I\right\|\|L(t)\|
\end{aligned}
$$

and, applying the mean value theorem [2, p. 11, Corollary 2]

$$
\begin{aligned}
\| t B- & t A-L(t)\|\leqq\| L \|(t) \\
& \cdot\left\{\sup _{0 \leqq \theta \leqq 1}\left\|\psi^{\prime}(a(t), \theta L(t))-\psi^{\prime}(a(t), 0)\right\|+\left\|\psi^{\prime}(a(t), 0)-I\right\|\right\} .
\end{aligned}
$$

Now it is clear that $\lim _{t \rightarrow 0} L(t)=0$ so that, using the continuity of $\psi^{\prime}$, this inequality implies that

$$
\lim _{t \rightarrow 0}\|B-A-(L(t) / t)\| /\|L(t) / t\|=0,
$$

and hence that

$$
\lim _{t \rightarrow 0}(L(t) / t)=B-A
$$

A similar use of the mean value theorem gives

$$
\|t C(s, t)-t A-s L(t)\| \leqq s\|L(t)\| \lambda(t)
$$

where $\lim _{t \rightarrow 0} \lambda(t)=0$ and, combining this with (3.1), we obtain the result stated.

Definition. An affine transformation of a system of paths on $M$ is a homeomorphism of $M$ onto itself which preserves the paths considered as parametrized curves. 
Let $\phi$ be an affine transformation and write $x^{\prime}=\phi(x)$ for $x \in M$. Let $A$ be any vector in $T_{x}$ and consider the path $\exp _{x} t A(t \geqq 0)$ issuing from $x$ with tangent vector $A . \phi\left(\exp _{x} t A\right)$ is a path issuing from $x^{\prime}$ and can thus be written uniquely as $\exp _{x^{\prime}} t A^{\prime}$ where $A^{\prime} \in T_{x^{\prime}}$. We define a mapping $\phi_{x}^{\prime}$ of $T_{x}$ into $T_{x^{\prime}}$ by putting $\phi_{x}^{\prime}(A)=A^{\prime}$. It is not difficult to see that $\phi_{x}^{\prime}$ is one-to-one, onto and positively homogeneous of degree one. Locally we have the formula

$$
\phi=\exp _{x^{\prime}} \phi_{x}^{\prime} \exp _{x}^{-1}
$$

and this equation, together with the homogeneity of $\phi_{x}^{\prime}$, implies that $\phi_{x}^{\prime}$ is a homeomorphism. We now prove

LEMMA 3.2. $\phi_{x}^{\prime}$ is a linear transformation.

Proof. Choose $C$-neighborhoods $U, U^{\prime}$ of $x, x^{\prime}$ so that $\phi(U) \subset U^{\prime}$ and consider those positive values of $t$ on two paths $\exp _{x} t A, \exp _{x} t B$ for which these paths lie in $U$. With our notation their images under $\phi$ are $\exp _{x^{\prime}} t A^{\prime}$ and $\exp _{x^{\prime}} t B^{\prime}$. Then, with the notation of Lemma 3.1, construct points $c(s, t), c^{*}(s, t)$ in $U, U^{\prime}$ respectively and vectors $C(s, t), C^{*}(s, t)$ in $T_{x}$ and $T_{x^{\prime}}$. Since $\phi$ is an affine transformation $c^{*}(s, t)=\phi(c(s, t))$ and $C^{*}(s, t)=\phi_{x}^{\prime}(C(s, t))$. Letting $t \rightarrow 0$ in the last equation we obtain, using Lemma 3.1 and the continuity of $\phi_{x}^{\prime}$, $\phi_{x}^{\prime}((1-s) A+s B)=(1-s) A^{\prime}+s B^{\prime}$ for $0 \leqq s \leqq 1$. This equation, together with the homogeneity of $\phi_{x}^{\prime}$, shows that $\phi_{x}^{\prime}$ is linear.

We can now prove

THEOREM 3.1. An affine transformation is a diffeomorphism.

Proof. Let $x$ be an arbitrary point of $M$ and choose $C$-neighborhoods $U, U^{\prime}$ of $x, x^{\prime}$ so that $\phi(U) \subset U^{\prime}$. Now choose a point $y$ in $U$ distinct from $x$. Then Lemma 3.2 together with the local formula

$$
\phi=\exp _{y^{\prime}} \phi_{y}^{\prime} \exp _{\nu}^{-1}
$$

shows that $\phi$ is a local diffeomorphism at $x$. We add the remark that $\phi_{x}^{\prime}$ is the differential of $\phi$ at $x$.

We can apply Theorem 3.1 to prove a theorem on Finsler manifolds generalizing Theorem 11.1 on p. 61 of [4]. For the purposes of our theorem a Finsler manifold is defined as in [5] but with the analyticity assumption replaced by a $C^{\infty}$ assumption. We define an isometry of $M$ to be a diffeomorphism of $M$ onto itself whose differential preserves the length of tangent vectors. On the other hand the Finsler manifold can be given a metric space structure [5], where the distance function is not necessarily symmetric. Then our theorem is 
THEOREM 3.2. A distance preserving transformation of $M$ onto itself is an isometry.

Proof. The geodesics of a Finsler manifold form a system of paths as defined in $\$ 2$. Using the arguments given at the top of p. 61 of [4] it can be shown that a distance preserving transformation is an affine transformation which preserves arc length, and consequently $\phi_{x}^{\prime}$ preserves the length of tangent vectors. Theorem 3.1 implies that $\phi$ is a diffeomorphism and the remark at the end of the proof of that theorem shows that $\phi$ is an isometry.

4. Lemmas. In $\$ 5$ we show that Theorem 3.1 holds for projective transformations of reversible systems of paths on manifolds of dimension greater than two. In the present section we give three lemmas which we need in our proof of this fact.

Let $V$ denote a real vector space of dimension $d$ and origin $O$, and let $\tilde{V}$ denote the space of nonoriented directions through $O . \tilde{V}$ is a real projective space of dimension $d-1$ and the canonical projection $\rho$ of $V-O$ onto $\tilde{V}$ is analytic and of rank $d-1$. Define a norm in $V$ by identifying $V$ with $R^{d}$ and using the standard norm in $R^{d}$. Then we state the following lemma the proof of which is omitted.

Lemma 4.1. Suppose that $X_{n}, Y_{n}$ are sequences of vectors in $V-O$ such that $\lim _{n \rightarrow \infty}\left(\left\|X_{n}-Y_{n}\right\| /\left\|Y_{n}\right\|\right)=0$. Then $\lim _{n \rightarrow \infty} \rho\left(X_{n}\right)$ exists if and only if $\lim _{n \rightarrow \infty} \rho\left(Y_{n}\right)$ exists, and the two limits are equal.

The next two lemmas concern a reversible system of paths defined on a differentiable manifold $M$.

Lemma 4.2. Suppose $a, b$ are two paths through $a$ point $x$ of $M$ and suppose $a_{n}, b_{n}$ are sequences on these paths lying in a $C$-neighborhood $U$ of $x$, and such that $\lim _{n \rightarrow \infty} a_{n}=x=\lim _{n \rightarrow \infty} b_{n}$. Let $c_{n}$ be a point on the path from $a_{n}$ to $b_{n}$ in $U$. Then, if the direction at $x$ of the path from $x$ to $c_{n}$ in $U$ approaches a limit as $n$ approaches infinity, this direction lies in the plane determined by the directions of $a, b$ at $x$. Further every direction in the plane can be obtained as such a limit.

Proof. We use the mapping $\psi(y, X)$ defined as in the proof of Lemma 3.1 but with the local section chosen so that $e_{1}(x)=A, e_{2}(x)$ $=B$ where $A$ and $B$ are tangent to $a$ and $b$ respectively. We write $a_{n}=\exp _{x} r_{n} A, b_{n}=\exp _{x} s_{n} B=\exp _{a_{n}} L_{n}, c_{n}=\exp _{x} C_{n}=\exp _{a_{n}} t_{n} L_{n}$ so that

$$
r_{n} A=\psi\left(a_{n}, 0\right), \quad C_{n}=\psi\left(a_{n}, t_{n} L_{n}\right), \quad s_{n} B=\psi\left(a_{n}, L_{n}\right)
$$

where $0 \leqq t_{n} \leqq 1$. Then 


$$
\begin{gathered}
\left\|s_{n} B-r_{n} A-L_{n}\right\| \leqq \\
+s_{n} B-r_{n} A-\psi^{\prime}\left(a_{n}, 0\right) L_{n} \| \\
+\left\|\psi^{\prime}\left(a_{n}, 0\right)-I\right\|\left\|L_{n}\right\|
\end{gathered}
$$

and, applying the mean value theorem,

$$
\begin{aligned}
& \left\|s_{n} B-r_{n} A-L_{n}\right\| \\
& \leqq\left\|L_{n}\right\|\left\{\sup _{0 \leqq \theta \leqq 1}\left\|\psi^{\prime}\left(a_{n}, \theta L_{n}\right)-\psi^{\prime}\left(a_{n}, 0\right)\right\|+\left\|\psi^{\prime}\left(a_{n}, 0\right)-I\right\|\right\} .
\end{aligned}
$$

Now it is clear that $\lim _{n \rightarrow \infty} L_{n}=0$ so that, using the continuity of $\psi^{\prime}$, this inequality implies that

$$
\lim _{n \rightarrow \infty}\left\|s_{n} B-r_{n} A-L_{n}\right\| /\left\|L_{n}\right\|=0
$$

and hence that

$$
\lim _{n \rightarrow \infty}\left\|s_{n} B-r_{n} A\right\| /\left\|L_{n}\right\|=1
$$

A similar use of the mean value theorem gives

$$
\begin{gathered}
\left\|C_{n}-r_{n} A-t_{n} L_{n}\right\| \leqq t_{n}\left\|L_{n}\right\| \lambda_{n}, \\
\left\|C_{n}-s_{n} B+\left(1-t_{n}\right) L_{n}\right\| \leqq\left(1-t_{n}\right)\left\|L_{n}\right\| \mu_{n},
\end{gathered}
$$

where $\lim _{n \rightarrow \infty} \lambda_{n}=0=\lim _{n \rightarrow \infty} \mu_{n}$. Now we put $Y_{n}=\left(1-t_{n}\right) r_{n} A+t_{n} s_{n} B$ and, using these inequalities, we have

$$
\left\|C_{n}-Y_{n}\right\| \leqq\left(1-t_{n}\right) t_{n}\left\|L_{n}\right\|\left(\lambda_{n}+\mu_{n}\right) .
$$

Then (4.1) and the obvious inequality

$$
t_{n}^{2}\left(1-t_{n}\right)^{2}\left(r_{n}^{2}+s_{n}^{2}\right) \leqq r_{n}^{2}\left(1-t_{n}\right)^{2}+t_{t_{n}}^{2} s_{n}^{2}
$$

show that

$$
\lim _{n \rightarrow \infty}\left(\left\|C_{n}-Y_{n}\right\| /\left\|Y_{n}\right\|\right)=0 .
$$

Lemma 4.1 now implies that, if $\lim _{n \rightarrow \infty} \rho\left(C_{n}\right)$ exists, then it is equal to $\lim _{n \rightarrow \infty} \rho\left(Y_{n}\right)$ which obviously lies in the plane determined by $\rho(A)$ and $\rho(B)$. Further, by choice of $r_{n}, s_{n}, t_{n}$ it is clear that one can arrange that $\lim _{n \rightarrow \infty} \rho\left(Y_{n}\right)$ exist and be equal to any direction in this plane. Lemma 4.1 shows that $\lim _{n \rightarrow \infty} \rho\left(C_{n}\right)$ is also equal to this direction. Thus Lemma 4.2 is proved.

Our next lemma is concerned with a mapping which generalizes 
bipolar coordinates. Let $\widetilde{T}_{x}$ denote the space of nonoriented directions tangent to $M$ at $x$ and define a local mapping of $M$ onto $\widetilde{T}_{x}$ by $\tau_{x}=\rho_{x} \exp _{x}^{-1}$, where $\rho_{x}$ is the canonical projection of $T_{x}$ onto $\widetilde{T}_{x}$. With the exception of the point $x$ itself $\tau_{x}$ is defined in a normal neighborhood of $x$ and is of class $C^{\infty}$. Now let $y, z$ be two points lying in a $C$ neighborhood $U$ and consider the $C^{\infty}$ mapping $\tau_{y z}=\tau_{y} \times \tau_{z}$ of $U-\{y, z\}$ into $\tilde{T}_{y} \times \tilde{T}_{z}$. We prove

LemmA 4.3. The rank of $\tau_{y z}$ is equal to $\operatorname{dim} M$ for points not on the path in $U$ through $y$ and $z$.

Proof. The kernel of the different $: v_{1} \tau_{y z}$ at a point $x \in U$ is the intersection of the kernels of the differentials of $\tau_{y}$ and $\tau_{z}$. Thus it consists of the vectors at $x$ which are tangent to both paths $x y, x z$ and can be nonzero only if $x$ is on the path through $y$ and $z$.

\section{The differentiability of projective transformations.}

DEFINITION. A projective transformation of a reversible system of paths on $M$ is a homeomorphism of $M$ onto itself which preserves the paths considered as point sets.

Let $\phi$ be a projective transformation of $M$ and write $x^{\prime}=\phi(x)$ for $x \in M$. We define a transformation $\tilde{\phi}_{x}$ of $\widetilde{T}_{x}$ into $\widetilde{T}_{x^{\prime}}$ in the following way. Let $\alpha$ denote a direction at $x$ and let $a$ be a path through $x$ with direction $\alpha . \phi a$ is a continuous curve in $M$ and, by hypothesis, its point set lies on a path through $x^{\prime}$. We define $\tilde{\phi}_{x}(\alpha)$ to be the direction of this path at $x^{\prime}$. It is not difficult to see that $\tilde{\phi}_{x}$ is well-defined, oneto-one and on to. To show that $\tilde{\phi}_{x}$ is continuous we first choose normal neighborhoods $U, U^{\prime}$ of $x, x^{\prime}$ so that $\phi(U) \subset U^{\prime}$. Then we construct a local analytic homeomorphism $\sigma_{x}$ of $\tilde{T}_{x}$ into $T_{x}$, defined near $\alpha$ with its image in $\exp _{x}^{-1}(U)$, and such that $\rho_{x} \sigma_{x}$ is the identity transformation. Then the formula

$$
\tilde{\phi}_{x}=\tau_{x^{\prime}} \phi \exp _{x} \sigma_{x}
$$

shows that $\tilde{\phi}_{x}$ is continuous. We now prove

Lemma 5.1. For $\operatorname{dim} M>2, \tilde{\phi}_{x}$ is induced by a linear transformation of $T_{x}$ onto $T_{x^{\prime}}$, and is thus analytic.

Proof. The lemma follows from the fundamental theorem of real projective geometry if we show that $\tilde{\phi}_{x}$ takes directions lying in a 2 -plane at $x$ into directions lying in a 2-plane at $x^{\prime}$. To do this, consider two directions $\alpha, \beta$ at $x$ and choose paths $a, b$ through $x$ with these directions. Let $\gamma$ denote a direction in the plane of the directions $\alpha, \beta$. Now choose $C$-neighborhoods $U, U^{\prime}$ of $x, x^{\prime}$ respectively 
so that $\phi(U) \subset U^{\prime}$ and, with the notation of Lemma 4.2, construct sequences $a_{n}, b_{n}, c_{n}$ so that the direction $\gamma_{n}$ of the path from $x$ to $c_{n}$ at $x$ approaches $\gamma$ as $n$ approaches infinity. Put $\gamma_{n}^{\prime}=\tilde{\phi}_{x}\left(\gamma_{n}\right)$ so that $\gamma_{n}^{\prime}$ is the direction at $x^{\prime}$ of the path from $x^{\prime}$ to $c_{n}^{\prime}=\phi\left(c_{n}\right)$ in $U^{\prime}$. Then, by the continuity of $\tilde{\phi}_{x}, \lim _{n \rightarrow \infty} \gamma_{n}^{\prime}$ exists and equals $\tilde{\phi}_{x}(\gamma)$. Thus, by Lemma $4.2, \tilde{\phi}_{x}(\gamma)$ lies in the plane of the directions $\tilde{\phi}_{x}(\alpha), \tilde{\phi}_{x}(\beta)$.

We can now prove

THEOREM 5.1. A projective transformation of a reversible system of paths on $M$ is a diffeomorphism if $\operatorname{dim} M>2$.

Proof. With our previcus : intations let $x$ be an arbitrary point of $M$ and choose $C$-neighborhoods $U, U^{\prime}$ of $x, x^{\prime}$ so that $\phi(U) \subset U^{\prime}$. Now choose points $y, z$ in $U$ so that $x$ does not lie on the path in $U$ through $y$ and $z$. Then $x^{\prime}$ does not lie on the path in $U^{\prime}$ through $y^{\prime}$ and $z^{\prime}$ and, because of Lemma $4.3, \tau_{y^{\prime} z^{\prime}}$ is a diffeomorphism of a neighborhood of $x^{\prime}$ onto a submanifold of $\widetilde{T}_{y^{\prime}} \times \widetilde{T}_{z^{\prime}}$. Thus on this submanifold $\tau_{y^{\prime} z^{\prime}}$ will have an inverse which we denote by $\sigma_{y^{\prime} z^{\prime}}$. Using Lemma 5.1 the local formula

$$
\phi=\sigma_{y^{\prime} z^{\prime}}\left(\tilde{\phi}_{y} \times \tilde{\phi}_{z}\right) \tau_{y z}
$$

shows that $\phi$ is a local diffeomorphism at $x$. We add the remark that $\tilde{\phi}_{x}$ is induced by the differential of $\phi$ at $x$.

\section{REFERENCES}

1. J. Douglas, The general geometry of paths, Ann. of Math. 29 (1927), 143-168.

2. S. Lang, Introduction to differentiable manifolds, Interscience, New York and London, 1962.

3. R. E. Trabor, A fundamental lemma on normal coordinates and its applications, Quart. J. Math. Oxford Ser. (2) 8 (1937), 142-147.

4. S. Helgason, Differential geometry and symmetric spaces, Academic Press, New York and London, 1962.

5. J. H. C. Whitehead, On the covering of a complete space by the geodesics through a point, Ann. of Math. 36 (1935), 679-704.

NORTHWESTERN UNIVERSITY AND

SOUTHAMPTON UNIVERSITY, ENGLAND 